\title{
Theory of Computation, Fuzziness and a physics of the immaterial
}

\author{
Marco Elio Tabacchi ${ }^{1}$ Settimo Termini $^{2}$ \\ ${ }^{1}$ DMI, Università degli Studi di Palermo and Istituto Nazionale di Ricerche Demopolis (Italy) \\ ${ }^{2}$ DMI, Università degli Studi di Palermo (Italy) and ECSC (Spain)
}

\begin{abstract}
In this paper we advance three clear-cut proposals as a contribution to the discussion on the role of notions of Computation and Fuzziness as a bridge between Hard and Soft Sciences. We suggest that an important difference between the two great families of science lies in their subject or research having a grounding in nature or not, and that Theory of Computation is a glaring exception to this classification, being a textbook hard science but dealing with the immaterial. We further advance that such unicity is strongly connected with Church-Turing thesis, and discuss about the role of Computation and Fuzziness as pillars of immaterial sciences
\end{abstract}

Keywords: Theory of Computation, Fuzziness, Church-Turing thesis

\section{Introduction}

Relationships between Hard and Soft Sciences have a long-standing and well studied history. We have previously discussed the role of Fuzzy Sets Theory as a methodological bridge between the seemingly disconnected worlds of humanities and science $[1,2]$. In this paper we debate a different aspect of such separation. Starting with a discussion on nomenclature of science in general and of Computer Science in particular, we show how the usual criteria for describing a separation of the two worlds in science can be shifted from the objects and methods of research to something more deep and ingrained: the relationship of the research domain with objective phenomena, as suggested by Nature. The classification criterion we offer is more clear-cut and much less ambiguous than the current ones used in the academic discussion, and has the definite advantage of being impervious to the continuous shifting in the treatment of precision by disciplines from both sides, with classic human sciences becoming day after day more attracted by the illusion of precise measurement, diffused metrics and the "everything is quantifiable" mantra, and at the same time the harder subjects lured by Cognitive Science in the very human world of imprecision, inexact reasoning and Fuzziness. Such classification leaves out a notable exception: Theory of Computation (along with the broader family of Computer Science), and so we discuss the implications of the existence of this anomaly, finding it a strength instead of a weakness and pinpointing a possible explanation. Recognizing that Fuzziness is a central part of the creative chaos that has characterized the birth and development of new scientific notions, we look for connections between the unicity of Theory of Computation and the power of representation and flexibility of Fuzziness.

The present works does not exists in isolation in respect to our previous research: the idea hereby sketchily presented is one logical step in a long stretch of discussion. A direct antecedent, where a first pass at the idea of the unicity of Computer Science along with some of the consequences on Fuzzy Sets Theory is given in [3]. The strong links, or the family resemblance term we have chosen for them, between FST and hard sciences have been explored in $[4,5]$. Some applications of this phenomenon relative to fuzziness can be found in $[6,7,8]$, while a different approach regarding network is detailed in $[9,10]$.

In the following section, we will try to clearly state what we mean with "physics of the immaterial", along with examples and a comparison between human and hard sciences. Section 3 will discuss with relationships and consequences of the core idea to Fuzziness and briefly explore the existing relationship between Computability and Fuzziness. Section 4 draws parallels between fuzziness and computing as sciences of the immaterial. A recap and some final remarks will conclude the paper.

\section{Material and immaterial in sciences}

\subsection{Disciplines and nomenclature}

As in many other scientific fields, what today is commonly labelled as Computer Science in reality goes by a myriad of different nomenclatures, depending on personal preferences, historical reasons, noble intents and perhaps less noble funding allocations. Just as an example of the many different terms, researchers dealing with the efficient transmission of information go under the Information Sciences label, while the community that deals with automation and controls has first veered toward the Cybernetic moniker, settling on the more sci-fi friendly Artificial Intelligence in present times; the more human-inclined have adhered to the $\mathrm{Cog}$ nitive Science bandwagon, while the holistic bunch 
has soldiered on the System Sciences designation; the more traditionalists have insisted on a revival of the Informatics label, and the avant-garde is working on different terms attached to the Computing suffix. Furthermore, all those names belong to the Computer Science family, but such categorization are not immutable in time or space, and no really strict boundaries are built (except for academic reasons): it is still not clear if Cybernetics is also a part of System Sciences [11], if there are real differences between AI and Cybernetics (apart from the catchy name), what subset of Cognitive Sciences AI is, and so on.

This parcelization of names is not a privilege of Computer Science: it can easily be found both in hard sciences - e.g. physics, with its innumerable research fields ranging from quantum mechanics to astrophysics - and in humanities: social sciences have seen a flourishing of sub-disciplines, often with differences that lie more in the eye of the beholder than in some tangible realm. But a reflection centered more on what keeps together all this denominations in one discipline and less on what differentiates them can lead us to a precise characterization of Computability, and Computer Science as an extension, as a unique discipline (or family thereof).

\subsection{Material and immaterial}

There are obviously many ways to differentiate between hard and human sciences [12, 13], and choosing one among so many is an active stance on how the chooser sees self in this classification, and a telltale of personal background, studies, preferences and so on. But we maintain that at least one of such differences is objective enough to be considered a sensitive enough discriminant: (In general) Hard Sciences deal with material, Humanities deal with immaterial. This proposition may at first glance seem quite peculiar and counterintuitive, and needs some clarification: we are not discussing here the devices used to discover facts and demonstrate theories in the respective fields, not the means to arrive at such discoveries, or the tools involved. The aim of any discipline is to uncover facts that are included into its boundaries (or in an intersection of borders when interdisciplinary research is in action) and by this to characterize and describe the components of such a territory. This is usually a process composed of repeated measures plus a lot of thinking, and tinkering with results, and philosophical/logical reasoning. In such a process precision of measurement is not always necessary, and sometimes even not desirable - something we have written at length about in [5]; despite this, a number of traditional soft sciences (two usual suspects among them are Sociology and Psychology) recently invested a lot of time and resources in a foundational shift from qualitative toward quantitative, with the aim of measuring persons (or social constructs) as physicists measure spins and energy, in the hope of becoming real sciences, an appalling use of terms, propagated by the same people who should know better how to avoid it. But even if one day, in an impossible turn of events, human sciences decide to abdicate completely the human factor, and solely concentrate on measurement, this could perhaps damage one of the most invoked distinction between Hard and Soft Sciences, but would not change a bit of the definition given here.

\subsection{Hard sciences deal with material...}

Proposition 2.2 in fact has nothing to do with the methods, and all with the objects. When we write that Hard Sciences deal with material we are highlighting how the object of scientific research is something real, existing in nature and there to be studied. Clearly hard sciences can (and should) be creative in their approach; formal models as removed from reality as is necessary can be described, measures and scales can contain as much imprecision as needed, abstractions and inventions can be freely employed to get to the most useful description. But in the end, the scope and the main ingredients are furnished to us - in some sense - by Nature itself. Physical properties such as velocity, position, charge can be described, measured and composed in many different ways, but as far as we know those are such crucial and evident parameters in a physical system just because they are suggested by such object of research; even if one day a truly Theory of Everything will come up, this would just let all the subdisciplines of physic coalesce in one, and finally an artificial nomenclature would be no more, but still inspiration for choices and experimentation would come from something material. This is even truer for disciplines that lie higher in humans' tree of knowledge, such as chemistry or biology: chemists have been proud for ages for having concocted materials that are not present in nature, new and useful inventions that demonstrate how ingenuity can go a long way toward overcoming the limits imposed by evolution, and the moment biologists have cracked the DNA code, their have aimed at (and at least in every simple case) creating new, original life combining elements from what was already known. But both these disciplines are invariably bound to the building blocks kindly provided by Nature itself: elements and their interactions for chemistry, biological structures and their interconnection for biology. The game such sciences are playing has a basic set or rules that matches with some objective extension of reality, and in this sense we call them material.

\section{4. ... Soft sciences with immaterial}

And Soft Sciences? After all, they are concerned with the most material things that we can think of, human beings and their social interaction. While this is true in a sense, the matter here lies in definitions. While Hard Sciences are clearly linked to a 
root in nature, the same is not true for humanities. Sociologist can dissect and discuss poverty (a material matter indeed), as psychologists can be concerned with prejudice and philosophers with ethics, but definitions for this term have no firm root in some natural phenomenon, like their hard counterparts. A concept such as poverty has no clear definition in itself, is very cultural and history-dependent, and a different way of characterizing it can completely change the meaning of research finding correlated to the concept itself. There is no clear way of anchor such concept as poverty, prejudice or ethics to anything really material, really ingrained in nature, and sometimes the impression given by Soft Sciences is that the object of the research, more than a description of the phenomenon itself, is a struggle between different position about whose definition is better suited to drive the correct conclusions. In this sense humanities deal with the immaterial: the research is aimed at finding good definitions for the phenomena, but the single phenomenon itself is abstract from reality, just an artificial $^{1}$ construction, and as such immaterial, as there is no direct correspondence between concept and implementation; one explicandum, as many explicata as needed by the context $[14,15]$, no grounding in the physical world - anyone is welcome to come and give a different definition, as long as it is non logic-defying. Immaterial indeed.

\section{The exception of computation}

For sure, Computer Science is commonly classified among Hard Sciences, as phenomena studied in its context are usually measurable, controllable and repeatable; furthermore, the object of research may appear to be the implementation of its theory, namely computing machines. Nevertheless, we maintain that computation sits in a special position with respect to Proposition 2.2: Theory of Computation is a Hard Science that deals with immaterial Once again, here lies an apparent contradiction in terms. What discipline can be more material than Computer Science, a human endeavor entirely and squarely aimed at building a machine? And this is exactly the point. It doesn't matter what the final realization is, being it a supercomputer, an AI robot or a multimillion nodes network: behind any and each of the possible, past present and future accomplishments of the discipline lies a theoretical layer of models, whose finesse is extreme and that is as removed from practical, material realization as it is humanly possible. In this view, Theory of Computation and Computer Science are a sort of counterparts of physics. As physics is a continue exercise in abstraction that nonetheless starts from a grounding in nature, in contrast Computation is the materialization in term of problem solvers, rea-

\footnotetext{
${ }^{1}$ Artificial in its straight sense, as in not occurring naturally.
}

soning machines and massive calculators of a fundamentally immaterial concept. Physics abstract from material, Computation materializes from an abstraction.

Is this exception to the rule just an accident of birth, or can we find a specific reason, rooted in the genesis of Computation and on its peculiarities, that render this anomaly inevitable? Our hypothesis is that different factors of historical origin concoct to the emergence in an already peculiar discipline of a central notion. Computer Science is the young science, born during the last century from a group of brilliant thinkers and scientists: is the science that looks for the answer to a problem never posed before in such terms and scale; it uses the theoretical fundaments and tools offered by mathematics and physics without being overwhelmed by them; and all of this around just one single, easy to describe and universal concept: Church-Turing thesis. The seventy five years-old thesis ${ }^{2}$ in its simplicity makes the whole discipline the precious gift of stability: whatever the different formalizations, different interpretations, different models, different technologies and different advancements we may think of, all such concepts and notions will be always related to the concept of Computability, and measured against it. Through Church-Turing thesis comes the singularity of a scientific discipline in which stable, strong and powerful theories can be constructed, phenomena formalized without having to resort to something properly material. In other words: we are confronted with the singular case of an informal notion which coincides with all the possible formalizations. A coincidence of the notions of explicandum and explicatum in Carnap's terminology. A unique case in the history of science. In our view, what makes "hard science" something "immaterial".

However the interpretation proposed and the role assigned is apparently different from the usual considerations done on this classical Thesis. From this point of view it is interesting to observe that - in the setting of information sciences (or in the terminology heralded here, in the setting of the "immaterial") a few central other notions (that do not possess the unicity of formalization, which seems to be a unicum of the notion of computation) like Kolmogorov complexity, became very interesting new scientific concepts (and the innovative concept is also the basis, the angular stone, of a new interesting theory), just after using some crucial results of the theory of computation. It is only the possibility of a Theory of Computabilty that allows us to transform into a universal, strong theory a very interesting family of intuitions and concepts, which without the strong theoretical support offered by the Church-Turing thesis support, would be deemed

${ }^{2} 2012$ is also the centennial of Alan M. Turing birthday. His seminal ideas are still capable to induce in people thinking along new paths, and some of is intuitions are still to bring the fruits they deserve. 
to remain only an interesting idea.

\section{Fuzziness and theory of computation}

What has the notion of Fuzziness to do with all this? As one of the most important cast members of the Theory of Computation play, through Fuzziness [16] we enrich the plot with the chance of modeling uncertainty and its synonyms [5, 17] (approximate and incomplete/inexact reasoning, partial knowledge and incomplete information, approximation and so on), and with it a new bridge toward a dialogue with humanities. To be more precise, Zadeh contribution - from the original idea [18] to the very recent Computing with Words paradigm shift $[19,20]$, provides a conceptual framework that can serve as a way to escape the sophisticated regimentation of such approaches as probability (in the Kolmogorovian axiomatization) and look at the presence of uncertainty in an innovative way.

Such conceptual freedom provides us with a (re)vision for many crucial topics in Theory of Computation. A complete list would not fit into these marginal notes, but just as an example, undoubtedly influenced by our personal biases and history:

- the introduction - into the previously purely combinatorial terrain of logic - of peculiar elements and tools culled from mathematical analysis, following the paths described by von Neumann, in order to generalize the notion of logical connective [21, 22]; while misconstruing Łukasiewicz's motivations, this new context allows to go beyond the limits of his original setting. We can clearly see this change of direction in the approach followed by Enric Trillas, starting from his pioneering work about the classification of "negations" $[23,24,25]$. In this view, there is no coincidence in Trillas been involved in reconsidering the validity of logical principles in this new and extended universe [26, 27];

- the possibility of establishing comparisons between probabilistic approaches and Zadeh's proposal. Despite these comparisons has a long history, it continues to be an interesting topic [28]. We must also consider connections with non-standard interpretations of probability: e.g. the one proposed by De Finetti $[29,30]$, and the attempts made by Coletti and Scozzafava [31]. It is worthwile to note that the connection between coherent conditional probability and fuzziness highlighted by Coletti and Scozzafava does not exhaust all the possibility offered by fuzzy set theory as they developed in the last decades;

- the introduction of a more flexible tool than Shannon's measure of information (Entropy) through the notion of measure of fuzziness. This new setting allows to introduce both more measures and more concep- tual interpretations $[32,33,34,35]$. Moreover, this helps in applying this notion not only in classical domains such as Pattern recognition, but also in new, peculiar ones like a quantitative approach to aesthetic theories [6, 15], in which straightforward application of classical Shannon theory produced ambiguous results according to scholars as Rudolf Arnheim [36]. Another interesting point is once again due to Zadeh. In fact, he has shown a completely new path of investigation, called Computing with Words [20], which poses the notion of fuzziness in strict connection with the roots of immaterial sciences - without forgetting the hypothesis done about the special role of computation in the setting of "immaterial sciences".

Let us go back to the notion of fuzziness. Why having the possibility of treating uncertainty in a very general way inside scientific theories is so important? Here we shall not review all the considerations provided by Lotfi Zadeh which in the field are well known to novice and experts alike . We limit ourselves to highlight just two points which deserve to be carefully scrutinized, postponing a complete and careful explanation at a later date :

- According to many biologists, error and imprecision is what allows change (and improvements!). Nobel laureate Rita Levi Montalcini titled one of her books "In praise of imperfection" [37];

- It has been noted that "observational predicates" cannot be "exact" (Rohit Parikh was one of the people supporting the argument in a very convincing way [38]). This observation poses many difficult questions - and this explains why it is not frequently afforded - but is also unavoidable from a theoretical point of view.

In our view this is sufficient enough to affirm two things. First: new innovative conceptual notions are crucial in the development of science (and this, shows the need for a constant interaction between technical, formal developments and philosophical reflection. Second: that both the notions of Computation and Fuzziness are crucial for a further development of information sciences (the reader can also choose another name in the list presented at the beginning, or outside it).

\section{Conclusions}

This paper can easily be summarized by looking at the propositions from the previous sections. We first stated what in our opinion is the most important difference between Hard and Soft Sciences, i.e.the field of action of the research: material in Hard Sciences, immaterial in Soft Sciences. Following that, we affirmed that Theory of Computation, 
in all its extensions that we commonly call Computer Science, constitutes an exception to the rule, as a quintessential Hard Science that nonetheless deals with immaterial. We then affirmed that the reason for such glaring exception should be looked for in the theoretical basis of computation, and more specifically in Church-Turing thesis. As a big finish, we dealt with the connections between this peculiarity of Computation and Fuzziness.

This paper is clearly the stub of a discussion, and opens many doors without even trying to close any. While we make a stand for the arguments in all propositions, we expect fierce critiques from the Soft Sciences researchers (especially of the younger generation), perhaps disagreeing on the "dealing with immaterial" proposition and being defined as removed from reality and battling on definitions more than on substance, as well as from the Hard Sciences camp, afflicted by the curse of exact measurement and possibly offended by the idea of natural limits to their topics of research.

The definitions we introduced here need more refinement, usually brought by time and discussions, plus a more varied class of examples from both Hard and Soft Sciences, in order to corroborate the hypothesis and perhaps graduate a bit more the much too binary classification imposed by Proposition 2.2. Additionally, the discussion on relationships between Fuzziness and Computation as pillars of immaterial sciences needs to be validated in a more rigorous way, and more connection points has to be found to give consistency to the theory. We are sure that presenting this topic at this venue will attract a pressing exchange of ideas and will allow us to define more clearly the points here presented, to add more useful examples to our arsenal and, in the general spirit of our research, also to find new connection between science and humanities.

\section{References}

[1] Settimo Termini and Marco Elio Tabacchi. Fuzzy set theory as a methodological bridge between hard science and humanities. submitted, 2012.

[2] Settimo Termini. On some "family resemblances" of fuzzy set theory and human sciences. In Rudolf Seising and Veronica Sanz, editors, Soft Computing in Humanities and Social Sciences, Studies in Fuzziness and Soft Computing. Springer, 2011.

[3] Settimo Termini. On fuzziness, its homeland and its neighbour. In Rudolf Seising, Enric Trillas, Claudio Moraga, and Settimo Termini, editors, On Fuzziness - A Homage to Lotfi A. Zadeh, Studies in Fuzziness and Soft Computing. Springer-Verlag, 2013.

[4] Marco Elio Tabacchi and Settimo Termini. Fuzziness and social life: informal notions, for- mal definitions. In Proceedings of NAFIPS 2012, 2012.

[5] Marco Elio Tabacchi and Settimo Termini. Varieties of vagueness, fuzziness and a few foundational (and ontological) questions. In Proceedings of EusFLAT 2011, Advances in Intelligent Systems Research, pages 578-583. Atlantis Press, 2011.

[6] Baingio Pinna and Marco Elio Tabacchi. A fuzzy approach to the role of symmetry in shape formation: The illusion of the scalene triangle. In Vito Di Gesù, Sankar Pal, and Alfredo Petrosino, editors, Fuzzy Logic and Applications, volume 5571 of Lecture Notes in Artificial Intelligence, pages 197-204. Springer Berlin-Heidelberg, 2009.

[7] Maurizio Cardaci, Vito Di Gesu, Maria Petrou, and Marco Elio Tabacchi. A fuzzy approach to the evaluation of image complexity. Fuzzy Sets Syst., 160(10):1474-1484, 2009.

[8] Maria Petrou, Marco Elio Tabacchi, and Roberta Piroddi. Networks of Concepts and Ideas. The Computer Journal, 53(10):17381751, 2010.

[9] Barbara Caci, Maurizio Cardaci, and Marco Elio Tabacchi. Come è piccolo il mondo in rete: un'ipotesi small-world sulla topologia di facebook. Sistemi Intelligenti, 3:451-458, 2010.

[10] Barbara Caci, Maurizio Cardaci, and Marco Tabacchi. Facebook as a small world: a topological hypothesis. Social Network Analysis and Mining, 2:163-167, 2012.

[11] Rudolf Seising. Cybernetics, system(s) theory, information theory and fuzzy sets and systems in the 1950 s and 1960s. Information Sciences, 180(23):4459 - 4476, 2010.

[12] Rudolf Seising and Veronica Sanz González. Introduction. In Soft Computing in Humanities and Social Sciences, Studies in Fuzziness and Soft Computing, pages 3-36. Springer, 2011.

[13] Rudolf Seising and Veronica Sanz González. Warren weaver's "science and complexity" revisited. In Soft Computing in Humanities and Social Sciences, Studies in Fuzziness and Soft Computing, pages 55-87. Springer, 2011.

[14] Rudolf Carnap. Logical Foundations of Probability. Chicago University Press, 1950.

[15] Marco Elio Tabacchi and Settimo Termini. Measures of fuzziness and information: some challenges from reflections on aesthetic experience. In Proceedings of WConSC 2011, 2011.

[16] Claudio Moraga. Towards a fuzzy computability? Mathware and Soft Computing, 6(23):163-172, 1999.

[17] Marco Elio Tabacchi and Settimo Termini. A few remarks on the roots of fuzziness measures. In Salvatore Greco, Bernadette Bouchon-Meunier, Giulianella Coletti, Mario Fedrizzi, Benedetto Matarazzo, and Ronald R. 
Yager, editors, Advances in Computational Intelligence, volume 298 of Communications in Computer and Information Science, pages 62 67. Springer Berlin Heidelberg, 2012.

[18] Lotfi A. Zadeh. Fuzzy sets. Information and Control, 8:338-353, 1965.

[19] Lotfi A. Zadeh. Fuzzy logic = computing with words. Fuzzy Systems, IEEE Transactions on, 4(2):103-111, 1996.

[20] Lotfi A. Zadeh. From computing with numbers to computing with words - from manipulation of measurements to manipulation of perceptions. International Journal of Applied Mathematics and Computer Science, 12:307$324,2002$.

[21] John von Neumann. The general and logical theory of automata. In Cerebral Mechanisms in Behavior the Hixon Symposium. J. Wiley, 1951.

[22] John von Neumann. Probabilistic logics and the synthesis of reliable organisms from unreliable components. In Claude E. Shannon and John MacCarthy, editors, Automata Studies. Princeton University Press, 1956.

[23] Claudi Alsina, Enric Trillas, and Llorenc J. Valverde. On some logical connectives for fuzzy sets theory. Journal of Mathematical Analysis and Applications, 93:15-26, 1983.

[24] Enric Trillas. Sobre funciones de negación en la teoría de subconjuntos difusos. Stochastica, 3(1):47-60, 1979.

[25] Enric Trillas. On negation functions in fuzzy set theory. In Senén Barro, Alberto Bugarin, and Alejandro Sobrino, editors, Advances in Fuzzy Logic, pages 31-43. Universidade de Santiago de Compostela, 1998.

[26] Enric Trillas. On exact conditionals. Stochastica, 13(1):137-143, 1992.

[27] Enric Trillas. Non-contradiction, excludedmiddle, and fuzzy sets. In Vito Di Gesu, S.K. Pal, and A. Petrosino, editors, Computer Science: Fuzzy Logic and Applications, volume 5571 of Lecture Notes in Computer Science, pages 1-11. Springer, 2009.

[28] Takehiko Nakama, Enric Trillas, and Itziar Garcia-Honrado. Axiomatic investigation of fuzzy probabilities. In Rudolf Seising and Verónica Sanz, editors, Soft Computing in Humanities and Social Sciences, volume 273 of Studies in Fuzziness and Soft Computing, pages 125-140. Springer, Berlin, 2011.

[29] Bruno de Finetti. La prévision: ses lois logiques, ses sources subjectives. Annales de l'Institut Henri Poincaré, 7:1-68, 1937.

[30] Bruno de Finetti. Theory of Probability, volume 1. John Wiley and Sons, New York, 1974.

[31] Giulianella Coletti and Romano Scozzafava. Probabilistic Logic in a Coherent Setting, volume 15 of Trends in Logic. Dordrecht: Kluwer, 2002 .
[32] Aldo de Luca and Settimo Termini. Entropy and energy measures of a fuzzy set. In Madan M. Gupta, Rammohan K. Ragade, and Ronald R. Yager, editors, Advances in Fuzzy Set Theory and Applications, pages 321-338. North-Holland, 1979.

[33] Aldo de Luca and Settimo Termini. Entropy measures in fuzzy set theory. In Madhusudan G. Singh, editor, Systems and Control Encyclopedia, pages 1467-1473. Pergamon Press, 1988.

[34] Enric Trillas and Teresa Riera. Entropies in finite fuzzy sets. Information Sciences, 15:159168, 1978.

[35] Enric Trillas and Claudi Alsina. Sur les mesures du degré de flou. Stochastica, 3(1):8184, 1979.

[36] Rudolf Arnheim. Entropy and Art. University of California Press, Berkeley, CA, 1969.

[37] Rita Levi-Montalcini. In Praise of Imperfection. Sound Writings, 1920.

[38] Rohit Parikh. The problem of vague predicates. In Language, Logic and Method, volume 31 of Boston Studies in the Philosophy of Science New York, N.Y., pages 241-261. 1983. 